\title{
Comparison of stretch reflexes and shortening reactions in activated normal subjects with those in Parkinson's disease
}

\author{
COLIN J. ANDREWS, ${ }^{1}$ PETER D. NEILSON, ${ }^{2}$ AND JAMES W. LANCE \\ From the Division of Neurology, The Prince Henry Hospital, and the School of Medicine, \\ University of New South Wales, Sydney, Australia
}

\begin{abstract}
SUMMARY The stretch reflexes and shortening reactions evoked in six normal subjects during reinforcement were compared with those recorded from patients with Parkinson's disease. Although the responses to stretch and shortening of triceps were similar in both groups, they differed in the biceps muscle. In Parkinson's disease increasing muscle stretch of biceps was associated with increasing reflex activity, whereas the reverse relationship occurred in the activated normal subjects. The biceps EMG during sinusoidal stretching comprised two peaks in the activated normal group, a phenomenon not seen in Parkinson's disease. It is postulated that this response in the activated normal group is due to activation or reinforcement engaging long-loop reflexes. It is concluded that the rigidity of Parkinson's disease is not simply an exaggeration of the stretch reflexes found in normal man but differs in that the effects of flexor reflex afferent nerve fibres are submerged by group Ia afferent activity, and that some long-loop reflex pathways are no longer operative in Parkinson's disease.
\end{abstract}

In the course of a previous investigation (Andrews, Burke, and Lance, 1973) it was noted that tense normal subjects with exaggerated physiological tremor had stretch and shortening reactions, features usually ascribed to Parkinson's disease and other disorders of the basal ganglia.

Tonic stretch reflexes have been described previously in normal subjects (Mark, 1963) and the similarities to Parkinsonian rigidity emphasized (Landau, Struppler, and Mehls, 1966). Reinforcement probably activates both alpha and gamma motoneurones (Gassel and Diamantopoulos, 1964) and may thus provide for short periods the 'increased fore brain drive' which becomes continuous in Parkinson's disease.

The reflex responses to muscle stretch and shortening have now been examined in normal subjects during reinforcement and compared with those recorded from patients with Parkin-

1 Edwin and Daisy Street Research Fellow in Neurology.

2 Centre Industries Research Scholar.

Address for reprints: Division of Neurology, The Prince Henry Hospital, Little Bay, N.S.W. 2036, Australia. son's disease to evaluate whether the rigidity of this disorder could result from disinhibition of normal activating or reinforcing mechanisms.

\section{METHODS}

The stretch reflexes and shortening reactions of the biceps and triceps muscles were examined in six normal subjects using the same techniques as described for the analysis of the stretch reflexes and shortening reactions in Parkinson's disease (Andrews, Burke, and Lance, 1972). In brief, the relationship of the reflex response to the velocity of stretch was determined by linear stretching and shortening movements over a range of velocities through a joint angle of $100^{\circ}-110^{\circ}$. The responses to changes in muscle length were determined by dividing the amplitude of movements into sequential steps of approximately equal amplitude, and also by using sinusoidal movements of small amplitude at different centres of oscillation. The electromyograph (EMG) produced by passive joint movement was recorded by pairs of surface electrodes $10 \mathrm{~cm}$ apart applied over the relevant muscles. The EMG potentials 
were monitored on an oscilloscope to exclude movement artefact and were integrated (time constant $0 \cdot 2 \mathrm{sec}$ ) to provide measures of the stretch reflex and the shortening reaction. Joint position was measured by a goniometer and also monitored on an oscilloscope, so that any joint position or amplitude of movement could be reproduced accurately. The goniometer output was differentiated (time constant $5 \mathrm{msec}$ ) to provide a voltage proportional to angular velocity. The velocity of joint movement, joint position, direct EMG, and integrated EMG were recorded on a four-channel type S Offner Dynograph or on a Grass polygraph.

The phase relationships of the reflex response during sinusoidal stretching were determined as described in full by Burke, Andrews, and Gillies (1971). The EMG potentials were amplified and full-wave rectified, and the responses to 20 stretching cycles were averaged by an Enhancetron fixedprogramme averaging computer which was triggered when the goniometer output exceeded a predetermined trigger voltage. Joint position was averaged simultaneously. The averaged reflex response to sinusoidal stretching was displayed on an oscilloscope and photographed with a Polaroid oscilloscope camera.

Reinforcement was obtained in normal subjects by asking the patient to perform difficult mental calculations, to clench one fist or to pull one hand against the other (Jendrassik manoeuvre), or to abduct the thighs forcibly.

\section{RESULTS}

LINEAR STRETCH AND SHORTENING IN ACTIVATED NORMAL SUBJECTS

STRETCH REFLEXES 1 . Biceps brachii The dynamic component of the biceps stretch reflex fluctuated markedly during activation, even though subjects were continually exhorted to maximum effort. The degree of reinforcement was not sufficiently constant and reproducible to permit calculation of the relationship between the dynamic stretch reflex and the velocity of stretch. When the response to muscle length was examined by systematically oscillating the limb at different joint positions, the dynamic stretch reflex was found to become maximal between $60^{\circ}$ and $80^{\circ}$ of flexion of the elbow joint, after which it was progressively reduced by further increases in muscle length (Fig. 1). The static component of the tonic stretch reflex fluctuated less than the dynamic stretch reflex during reinforcement and was diminished during the

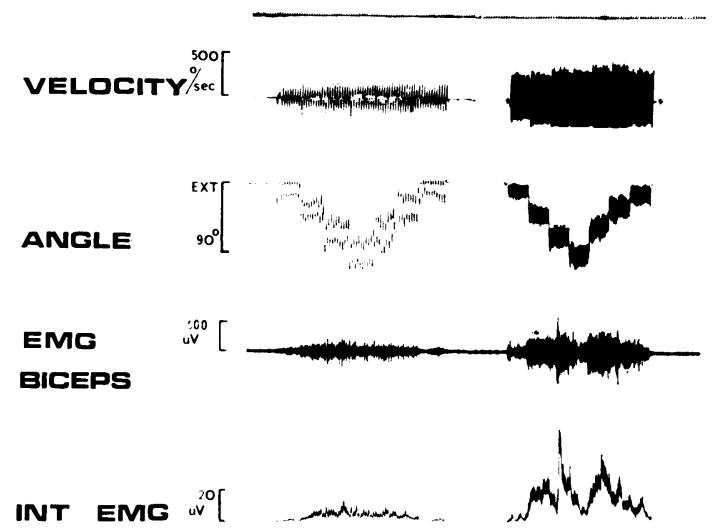

FIG. 1. The response of the dynamic stretch reflex of biceps to changes in muscle length in a normal subject during reinforcement. The electromyographic response tends to be maximal in a position between $60^{\circ}$ and $90^{\circ}$ of elbow flexion.

last phases of muscle stretch in the same way as the dynamic stretch reflex.

2. Triceps The triceps dynamic stretch reflex was more easily evoked than the biceps dynamic

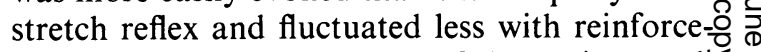
ment. The static component of the tonic stretch $\overrightarrow{0}$ reflex of triceps was less prominent than in biceps. Both dynamic and static components of the triceps stretch reflex were facilitated by increasing muscle length (Fig. 2).

SHORTENING REACTIONS Both dynamic and static shortening reactions were more conspicuous in the triceps than in the biceps. Commonly the dynamic shortening reaction was of greater amplitude than the dynamic stretch reflex (Fig. 2).

LINEAR STRETCH AND SHORTENING IN PARKINSON'S DISEASE

In triceps brachii, the dynamic stretch reflex of Parkinson's disease increased in proportion to the velocity of stretch in all instances, as reported previously (Andrews et al., 1972). This relationship was not usually apparent in biceps brachii. In both biceps and triceps, dynamic and static components of the stretch reflex were facilitated by increasing muscle length.

An involuntary contraction of biceps and tri- 

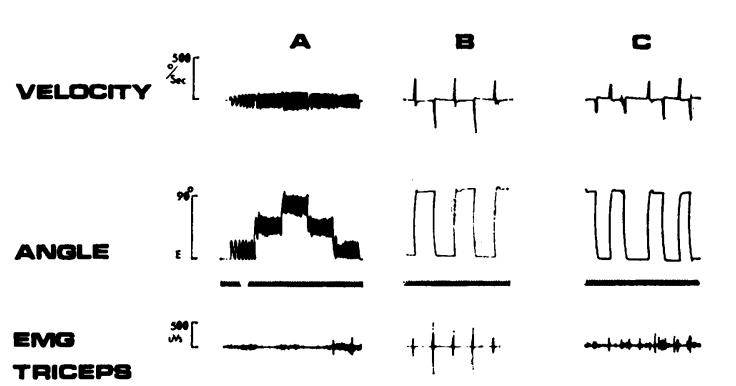

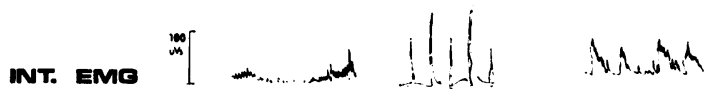

MILD

ceps was recorded during passive shortening of each muscle (dynamic shortening reaction) but sustained activity in the shortened position (the static shortening reaction) was inconstant.

\section{AVERAGED RESPONSES TO SINUSOIDAL STRETCH} NORMAL SUBJECTS DURING ACTIVATION The stretch reflexes and shortening reactions of biceps and triceps muscles were examined during sinusoidal stretch at $1 \mathrm{~Hz}$ through $0^{\circ}-90^{\circ}$ of elbow flexion in three normal male subjects during activation. The analysis of the biceps stretch reflex revealed two peaks, one with a phase lead greater than $90^{\circ}$ and one less than $90^{\circ}$. The two peaks were always present whatever the method of activation (Fig. 3). The triceps stretch reflex consisted of a single peak, the phase lead of which varied from $0-30^{\circ}$.

A response to sinusoidal shortening was recorded from the biceps muscle in two of the three subjects and from the triceps muscle in all subjects. The response of the triceps muscle to sinusoidal shortening was always a single peak with the phase lead being $180^{\circ}-200^{\circ}$ in relation to the most stretched position.

PATIENTS WITH PARKINSON'S DISEASE The stretch reflex of both biceps and triceps brachii consisted of a single peak in the EMG tracing. The phase lead of the stretch reflex was $0^{\circ}-45^{\circ}$ in biceps and $5^{\circ}-30^{\circ}$ in triceps in relation to the most stretched position. The stretch reflexes of

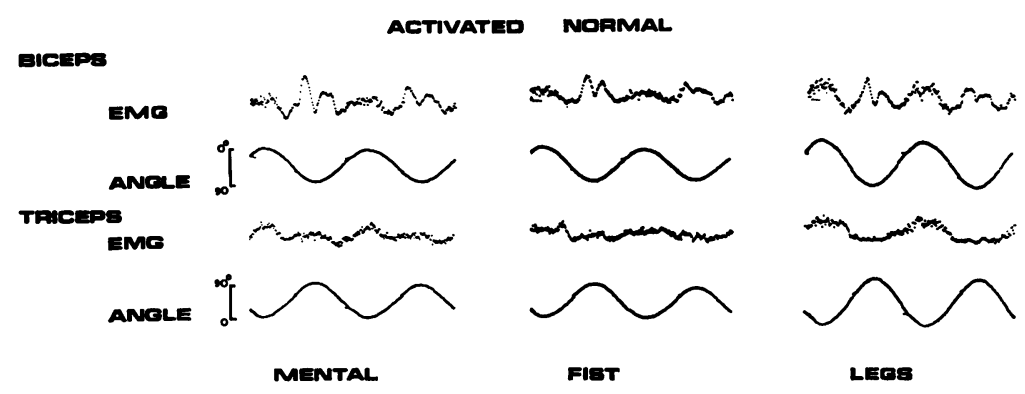

FIG. 3. The response of the stretch reflexes and shortening reactions during sinusoidal stretch of biceps and triceps, showing the similarity of response irrespective of the method of activation. Two peaks of electromyographic activity occur during sinusoidal stretch of biceps at $1.0 \mathrm{~Hz}$. Mental = mental activation produced by difficult calculations. Fist $=$ reinforcement by making a fist on the contralateral side. Legs= reinforcement by forceful abduction of both legs. 


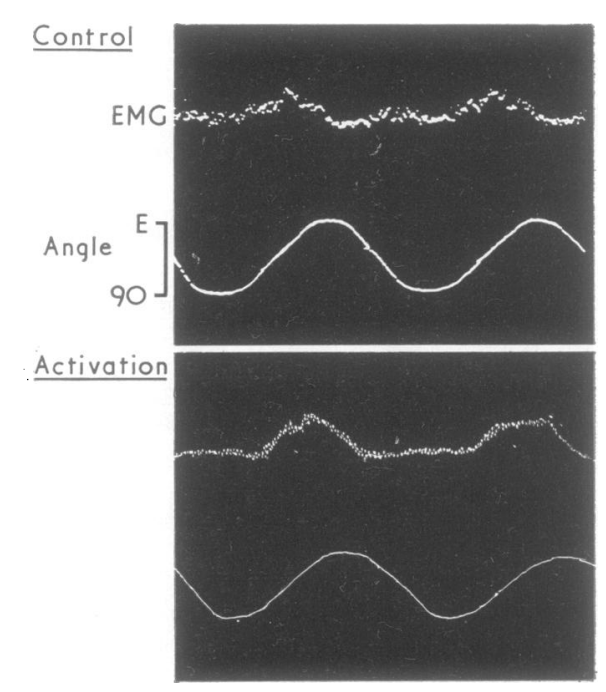

FIG. 4. The averaged electromyographic response to sinusoidal stretching at $1.0 \mathrm{~Hz}$ of the wrist flexors of a patient with Parkinson's disease at rest and during activation. The electromyographic response is augmented during activation and a single peak occurs only during stretching.

wrist flexor and extensor muscles were similar to those of biceps and triceps (Fig. 4).

\section{DISCUSSION}

Landau, Struppler, and Mehls (1966) compared the electromyographic responses of biceps and triceps to passive stretch and shortening in normal subjects activated by adrenaline injections and chilling to those patients with Parkinson's disease at rest. In activated normal patients, electromyographic activity was recorded during stretch and shortening in both muscles and, as chilling progressed, 'increasingly strong bursts of tonic activity occur in the brachialis at the point of maximal elbow flexion'. Landau et al. (1966) pointed out the similarities between their patients and activated normal subjects and concluded that segmental mechanisms are similar in both conditions. However, they found in some patients that the 'tonic brachialis discharge tends to be greater with stretch', quite unlike the responses elicited in activated normal subjects both in their study and our own.

The stretch reflex elicited from patients with
Parkinson's disease in response to sinusoidal movement demonstrated only one peak of EMG activity during stretching, with a phase lead of less than $45^{\circ}$. In contrast, the EMG of biceps in the activated normal group displayed two peaks during muscle stretch, some peaks having a phase lead greater than $90^{\circ}$. The response to sinusoidal stretch in the patients with Parkinson's disease can be explained on a segmental basis if one assumes that activity of group Ia afferent nerve fibres predominated over any influence exerted by Golgi tendon organs or secondary spindle endings. On the other hand, the double peak of the biceps EMG in normal subjects is difficult to explain solely by segmental mechanisms. Neilson (1972) has shown that the tonic stretch reflex of biceps in intact man during voluntary contraction contains a number of peaks of resonance and that the phase lead advances from $100^{\circ}$ to $180^{\circ}$ as the frequency of sinusoidal stretching increases towards $5.0 \mathrm{~Hz}$. $\mathrm{He}$ points out that a phase lead exceeding $90^{\circ}$ cannot be due to segmental events alone as oneo would then expect a decrease from $180^{\circ}$ towards

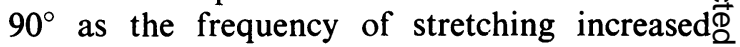
because of the higher velocity-sensitive component. He concluded that segmental and long loop reflex pathways probably interreact undero these conditions. The method of activation in this study could also introduce long loop? reflexes by the same means as voluntary muscle contraction reflexes. The fact that the EMG does not follow the full $360^{\circ}$ of the stretching cycle suggests that reinforcement activates the stretch reflex arc only partially and that anterior horn cells fire only during intervals of maximum reflex excitation. The two peaks in the biceps EMG can be interpreted as the response of partially activated parallel pathways of different latencies. If the level of activation is increased so that each pathway can follow the full $360^{\circ}$ of the sinusoidal cycle, then the EMG response can become sinusoidal but will have an amplitude and phase difference from that of the stretching motion. Thus the differences between the sinusoidal responses during sustained voluntary contraction reported by Neilson (1972) and the double peak EMG response during reinforcement reported in this paper could be due to the relative level of activation of polysynaptic pathways. 
This study has demonstrated that the stretch reflex in biceps brachii of normal man is inhibited by increasing muscle length, whereas that of triceps brachii is augmented by increasing muscle length. This situation appears analogous to the length-dependent inhibition found in the quadriceps and the length-dependent facilitation found in the hamstrings of spastic man (Burke, Gillies, and Lance, 1970, 1971). The differing effects of muscle length on antagonistic muscle pairs is best explained by the action of group II afferent nerve fibres and implies that biceps brachii, being the antigravity muscle of the upper limb in man, is inhibited by the so-called 'flexor reflex afferents'. The biceps stretch reflex is not inhibited by increasing muscle length in Parkinson's disease, or in spasticity (Ashby and Burke, 1971), probably because the effect of length is submerged by the increased activity in group Ia afferent fibres. This hypothesis is supported by the observation of Andrews et al. (1972) that ischaemia, which preferentially blocks the large group Ia afferent fibres, unmasks lengthdependent inhibition of the biceps brachii in patients with Parkinson's disease.

The authors wish to thank Mr. and Mrs. Edwin Street and Centre Industries for their financial support. The Figures were photographed by the
Department of Medical Illustration, University of New South Wales.

\section{REFERENCES}

Andrews, C. J., Burke, D., and Lance, J. W. (1972). The response to muscle stretch and shortening in Parkinsonian rigidity. Brain, 95, 795-812.

Andrews, C. J., Burke, D., and Lance, J. W. (1973). The comparison of tremors in normal, Parkinsonian and athetotic man. Journal of Neurological Sciences. (In press.)

Ashby, P., and Burke, D. (1971). Stretch reflexes in the upper limb of spastic man. Journal of Neurology, Neurosurgery, and Psychiatry, 34, 765-771.

Burke, D., Andrews; C. J., and Gillies, J. D. (1971). The reflex response to sinusoidal stretching in spastic man. Brain, 94, 455-470.

Burke, D., Gillies, J. D., and Lance, J. W. (1970). The quadriceps stretch reflex in human spasticity. Journal of Neurology, Neurosurgery, and Psychiatry, 33, 216-223.

Burke, D., Gillies, J. D., and Lance, J. W. (1971). Hamstrings stretch reflex in human spasticity. Journal of Neurology, Neurosurgery, and Psychiatry, 34, 231-235.

Gassel, M. M., and Diamantopoulos, E. (1964). The Jendrassik maneuver. 1. The pattern of reinforcement of monosynaptic reflexes in normal subjects and patients with spasticity or rigidity. Neurology (Minneap.), 14, 555560 .

Landau, W. M., Struppler, A., and Mehls, O. (1966). A comparative electromyographic study of the reactions to passive movement in parkinsonism and in normal subjects. Neurology (Minneap.), 16, 34-48.

Mark, R. F. (1963). Tonic stretch reflexes in the calf muscles in normal human subjects. Nature, 199, 50-52.

Neilson, P. D. (1972). Frequency response characteristics of the tonic stretch reflexes of biceps brachii muscle in intact man. Medical and Biological Engineering, 10, 460-472. 